\title{
Review of: "Cell region fingerprints enable highly precise single-cell tracking and lineage reconstruction"
}

\author{
Sébastien Tosi
}

Potential competing interests: The author(s) declared that no potential competing interests exist.

The authors first describe a new metric to blindly assess the validity of object assignments during object tracking (CRF), optimize its underlying parameters, and prove its robustness for varying cell types and imaging modalities. Next, they introduce a classical tracker (dubbed $\operatorname{Track}^{\mathrm{X}}$ ) relying on a basic set of object features (position, shape, orientation) to build the assignment cost matrix. They show that when assisted by CRF this tracker matches or over performs both generic and state of the art trackers for the task of tracking yeasts in YIT. To further improve inheritance robustness, they complement $\operatorname{Track}^{\mathrm{X}}$ by a set of adhoc features and division cues (overlap, symmetry, area) tailored to three challenging tracking problem. This final version is used to revisit known yeast biology and leads to some new insight.

\section{General comments}

I can only praise the high quality of the research, methodology and its presentation. Cell fingerprinting as a framework can certainly nicely complement existing trackers both to efficiently guide users to potential errors during validation, or to improve results by automated relinking. Track ${ }^{\mathrm{X}}$ is also a nice addition to the existing trackers, especially for the tracking of yeast cells.

However, in my opinion the paper slightly suffer from schizophrenia, being pulled toward the description of cell fingerprinting (generic) and the complex details of $\operatorname{Track}^{X}$ (specific). I would recommend the authors to better split these two parts, maybe by moving $\operatorname{Track}^{\mathrm{X}}$ description to supplementary, or even by splitting the content into two articles. In the first part, it would actually be interesting to assess how CRF improves the performance not only of $\operatorname{Track}^{X}$ but also of the other trackers, as well as how efficient it is in identifying their specific erroneous links (as opposed to artificial random errors). For assisted manual validation, reporting the fraction of truly erroneous links found when scanning the links from lowest to highest CRFs would give an idea on specificity and potential speedup during manual validation (as compared to going through all the links manually).

An extension to 3D would be interesting and should minimally be touched upon in the discussion, either by relying on 3D DCT, or more simply by extracting medial slice of the objects to fingerprint them. To facilitate this process, the software would probably benefit from being more modular and fully pluggable. For this, I 
would suggest an independent CRF module taking as input a pair of label masks (tracker output) and input images, and enabling navigation to low CRF links, correction and auto-correction.

\section{Specific comments}

- I wonder if rescaling (size information lost) is more efficient than using a fixed size window accommodating the largest observable object. Rescaling benefit should be better motivated.

- DCT is certainly cheap and efficient but have the authors been considering other compact image transformations such as SVD and Karhunen-Loève? Or even simple intensity correlation?

- Would masking surrounding objects before computing the DCT improve CRF discrimination power? Discontinuities should not show up in the first DCT coefficient and masking the correct object around invalid objects should be beneficial.

- It is difficult to see what the authors suggest in Figure 1. I would recommend removing subfigure $\mathbf{e}$ and focusing on a sub-region mostly displaying the problematic links in subfigures a-d.

- A better caption for Figure 3 would probably be "CRF performance (F-Score) and number of explored neighbors as a function of cell types and imaging settings". I would also rather set the dotted cyan lines at $2 x$ mean cell diameter to more clearly show that it falls close to performance neck. In subfigure $\mathbf{b}$, CRF frequency $\rightarrow$ frequencies.

- Figure 4: I would recommend to change caption to "Trackers performance (F-score) and CRF False Positive links miss rate as a function of neighborhood threshold".

- Line 49: I believe that DCT actually captures both luminance and contrast (and that both are useful features for fingerprinting!).

- Line 99: It would be interesting to vary the error fraction $(1 \%, 2 \%, 5 \% \ldots)$ to study if/when the method breaks, setting a baseline for when it is applicable. Details should also be added on the way that the errors were introduced. I believe that re-linking was not fully randomly (as suggested) but that randomly selected links where re-connected to neighbors (closest?).

- Line 108: If I am not wrong, it is not explicitly stated how the $f_{q}$ DCT coefficients were selected. I believe that they are the first 10 excluding the DC coefficient. 
- Line 125: Wouldn't "shape" or "aspect ratio" be more accurate than "size" for the basic set of features. Size is somehow equivalent to area (introduced as specific feature for mammalian cells).

- Line 315: Is the fixed perimeter set from the centroid of the objects? If so state it.

\section{Minor errors/imprecisions}

Abstract:

- Fourier $\rightarrow$ DCT

- Moving cells $\rightarrow$ quickly moving cells (a bit strange to set moving cells as a challenge for tracking!)

Line 23: Tracking can actually be done in a single step (without segmentation) so this is not fully correct

Line 40: with 1\% error rate: state that you talk about link error, not track/lineage error

Line 53: kinetic proofreading: I am not familiar with this adjective in this context

Imaging frequency (several times) $\rightarrow$ frame rate (frequency can be confusing in the context of the article) 